\title{
The Current State of Nuclear Medicine Physics Training: Findings of the AAPM/SNMMI Task Force
}

\author{
Beth A. Harkness ${ }^{1}$ and Frederic H. Fahey ${ }^{2}$ \\ ${ }^{1}$ Department of Radiology, Henry Ford Hospital, Detroit, Michigan; and ${ }^{2}$ Division of Nuclear Medicine and Molecular Imaging, \\ Department of Radiology, Boston Children's Hospital, Boston, Massachusetts
}

$\mathbf{T}$ he Society of Nuclear Medicine and Molecular Imaging (SNMMI) and the American Association of Physicists in Medicine (AAPM) decided in 2012 to establish a joint task force (Table 1) to review the state of nuclear medicine physics training and assess how to improve future training opportunities. The mission of the task force was to assemble a group of stakeholders in nuclear medicine and medical physics to estimate the demand for board-certified nuclear medicine physicists over the next 5-10 years, identify issues critical to supplying an adequate number of appropriately trained nuclear medicine physicists, and identify possible ways to facilitate that training.

The chair of the task force was active within both the SNMMI and the AAPM. Other members of the task force included representatives from the SNMMI, the AAPM, the American Board of Science in Nuclear Medicine (ABSNM), the American Board of Radiology (ABR), and the Commission for the Accreditation of Medical Physics Educational Programs (CAMPEP). The task force first met in person in July 2012. Over the next 2 years, the members met regularly both face to face and through conference calls, as well as communicating by email.

In 2014 the task force delivered its final report, which was approved by the boards of directors of both organizations and was recently published in the Journal of Applied Clinical Medical Physics (1). Here we summarize the findings, but readers are encouraged to review the full report.

Since the earliest days of nuclear medicine, the physicist has been an essential member of the developmental and clinical nuclear medicine team. The construction and implementation of nuclear medicine instruments and the development of clinical protocols has relied on collaboration between physicians and physicists. The nuclear medicine physicist has a strong understanding of physics and physiology, is an expert in instruments for measuring and imaging radiopharmaceuticals and in dosimetry for diagnostic and therapeutic procedures, and is responsible for devising and maintaining a quality assurance program for all imaging and nonimaging nuclear medicine equipment, including hybrid devices that incorporate CT or MR into a PET scanner or $\gamma$-camera. It is essential that the nuclear medicine physicist have a basic understand-

Received Dec. 15, 2015; revision accepted Feb. 10, 2016.

For correspondence or reprints contact: Beth A. Harkness, Department of Radiology, Henry Ford Hospital, 2799 W. Grand Blvd., Detroit, MI 48202.

E-mail: bethh@rad.hfh.edu

Published online Mar. 10, 2016.

COPYRIGHT (c) 2016 by the Society of Nuclear Medicine and Molecular Imaging, Inc.

DOI: $10.2967 /$ jnumed.115.171355 ing of physiology and molecular processes because of the functional nature of nuclear medicine, as well as knowing the physical and dosimetric aspects of radionuclide therapy. Although the well-trained diagnostic medical physicist may have a good basic understanding of nuclear medicine physics and may have a skill set overlapping that of the nuclear medicine physicist, nuclear medicine physics includes unique and essential aspects that may not be routinely covered within diagnostic imaging training.

The task force reviewed the current workforce of nuclear medicine physicists within the United States, using sources such as the membership database of the SNMMI, the certified medical physicist database of the Conference of Radiation Control Program Directors, and a 2012 professional survey by the AAPM. This review found that there were about 350-450 nuclear medicine physicists in the United States and that they composed less than $10 \%$ of the total number of medical physicists. It was estimated that fewer than 60 individuals classified themselves as primarily a nuclear medicine physicist.

Two organizations certify nuclear medicine physicists in the United States: the ABR and the ABSNM. To receive ABR certification, potential diplomates are required to pass a 3-part examination. Before allowing them to sit for the examination, the ABR reviews their experience and training, which, since 2014, has included completion of a medical physics residency. The ABR also requires participation in its maintenance-of-certification program. Thirty-seven medical physicists were certified in nuclear medical physics by the ABR between 2010 and 2014. To receive ABSNM certification in nuclear physics and instrumentation, candidates must pass a 2-part examination after meeting certain training and experience requirements but are not required to complete a nuclear medical physics residency. In addition, on January 1, 2015, the ABSNM established a maintenance-of-certification policy. Thirty-four physicists were certified in nuclear physics and instrumentation by the ABSNM between 2010 and 2014.

Because of the small number of currently certified nuclear medicine physicists, there is a critical need for CAMPEP-accredited residencies for clinical training in nuclear medicine physics. The models for medical physics residencies include 2-year programs in nuclear medicine physics, completion of an additional year in nuclear medicine physics after an imaging medical physics residency, and completion of a doctoral program in medical physics. There is also a hub-and-spoke model in which a central site administers the program and the residents receive instruction at associated sites. The CAMPEP website lists 11 residency programs for imaging medical physics, and these provide 10-15 residency slots within North America. No residencies exist that are specifically for 
TABLE 1

Members of the AAPM/SNMMI Task Force

\begin{tabular}{lll}
\hline \multicolumn{1}{c}{ Member } & Organization & \multicolumn{1}{c}{ Place of business } \\
\hline Beth A. Harkness, Chair & AAPM/SNMMI & Henry Ford Hospital, Detroit, Michigan \\
Jerry D. Allison & ABR & Georgia Regents University, Augusta, Georgia \\
Jessica B. Clements & AAPM & California Permanente Medical Group, Los Angeles, California \\
\hline Charles W. Coffey & CAMPEP & Vanderbilt University, Nashville, Tennessee \\
\hline Frederic H. Fahey & SNMMI & Boston Children's Hospital, Boston, Massachusetts \\
\hline Dustin A. Gress & AAPM & M.D. Anderson Cancer Center, Houston, Texas \\
\hline Paul E. Kinahan & & University of Washington, Seattle, Washington \\
\hline Edward L. Nickoloff & AAPM & Columbia University, New York, NY \\
\hline Osama R. Mawlawi & ABSNM & M.D. Anderson Cancer Center, Houston, Texas \\
Robert D. MacDougall & & Boston Children's Hospital, Boston, Massachusetts \\
Robert J. Pizzuitello & AAPM & Upstate Medical Physics, Victor, New York \\
\hline
\end{tabular}

nuclear medicine physics. However, several imaging residencies allow students to concentrate on nuclear medicine physics after the first year of a 2-year program, making them eligible to sit for the ABR certification examination.

To encourage new or existing imaging residencies to expand their programs to include nuclear medicine physics, the SNMMI awarded 2 grants - one in 2014 and another in 2015-each of which supported the training of a nuclear medicine physics resident. Building on the success of that program, the AAPM and SNMMI have partnered on a nuclear medicine residency program in 2016 that will support 2 programs for 2 years. Details are available on the AAPM (www.aapm.org) and SNMMI (www.snmmi. org) websites.

After completing its evaluation of the training needs of nuclear medicine physicists, the task force made several recommendations. The first was that its final report should serve as a guide for future efforts and, minimally, should be distributed to the relevant professional societies (AAPM, SNMMI, and Canadian Organization of Medical Physicists), the certifying bodies (ABR, ABSNM, CAMPEP, American Board of Medical Physics, and Canadian College of Physicists in Medicine), and the accreditation bodies (American College of Radiology, Intersocietal Accreditation Commission, and Joint Commission). The report should also be made widely available to interested individuals, perhaps through the websites of these organizations. Second, all certifying bodies for nuclear medicine physics should incorporate a formal residency and a maintenance-of-certification program as requirements for board certification. Because the task force reviewed data on only the current state of the field, it could not evaluate future needs. Therefore, a third recommendation was that a committee should be formed to evaluate these future needs, perhaps under the workforce assessment committee of the AAPM. The final recommendation was that relevant professional organizations should continue to fund residencies in nuclear medicine physics to encourage new and established imaging residencies to incorporate nuclear medicine physics training.

These recommendations were based on a combination of several factors: the high median age of current nuclear medicine physicists, the poor understanding of the training and certification processes, and the shortage of formal training programs.

Professional organizations such as the AAPM and, most importantly, the SNMMI must continue to support the training of nuclear medicine physicists and must continually reevaluate the needs of the field as the clinical and professional environment changes. To remain vital and progressive, nuclear medicine requires well-trained medical physicists to meet the complex requirements of quality patient care and to advance the field. A lack of qualified nuclear medicine physicists will have a negative impact on nuclear medicine as a whole.

\section{REFERENCE}

1. Harkness BA, Allison JD, Clements JB, et al. AAPM/SNMMI joint task force: report on the current state of nuclear medicine physics training. J Appl Clin Med Phys. 2015;16:5661. 